\title{
Attachment styles and the development of psychopathology
}

\author{
Carla Alves Pereira ${ }^{1}$, Bruna Melo $^{1}$, David Teixeira ${ }^{1}$, Alberto Marques ${ }^{1}$, Ana Lúcia Costa ${ }^{1}$, João \\ Brás $^{1}$, Rui Sousa ${ }^{1}$, Alzira Albuquerque ${ }^{1}$, Tânia Casanova ${ }^{1}$ \\ 'Department of Psychiatry and Mental Health, Centro Hospitalar Tondela-Viseu, EPE., Viseu, Portugal
}

BACKGROUND AND AIMS: Attachment style has been established to be a persistent framework for approaching and interpreting interpersonal relationships because, from birth, we immediately begin forming our attachment styles. Thus, it can be inferred that attachment has the ability to affect an exceptional amount of our interpersonal and mental health experience. Early patterns of infant attachment have been shown to be an important influence on adult social behaviour, affect regulation, temperament traits and the occurrence of psychopathology. These attatchment syles are divided into secure, anxious and avoidant, with the latter two representing insecure attachment.

OBJECTIVES AND METHODS: To present a non-systematic review of the literature about the early attachment experiences and

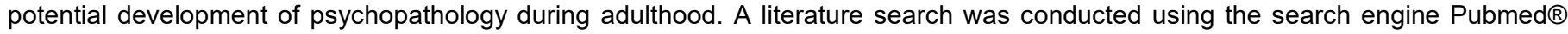
and the keywords attachment styles, psychopathology.

\section{RESULTS}

There is evidence in the literature confirming a significant link between adverse early attachment experiences and the development of psychopathology in adolescence and adulthood ${ }^{1}$. Or otherwise, research into attachment theory has yielded data suggesting that insecure attachment styles increase the reporting likelihood of mental aberrance, whereas secure attachment styles correlate with better mental health².

Research on attachment and personality disorders has
demonstrated that insecure attachment style, although not
being linked to specific personality disorders per se,
predispose a person to certain personality traits and
interpersonal conflicts that can, in turn, develop into a
personality disorder ${ }^{3}$.

Anxious attachment is more associated with traits such as neuroticism and emotional dysregulation; and avoidant attachment, with inhibitedness ${ }^{3}$.
An insecure adult attachment style almost universally was associated with a higher likelihood of having a mood or anxiety disorder ${ }^{2}$

Eating disorders and attachment have also been thoroughly researched and shown to be associated with attachment insecurity ${ }^{2}$.

An insecure attachment style was associated with an increased likelihood of reporting suicidal ideation and suicide attempts 2,4

Somehow, this development can be justified by some suggestions that patterns of early attachment influence brain development, contributing to permanent alterations in neural structure (animal studies) ${ }^{5}$, and proposes that childhood adversity causes early activation of the hypothalamic-pituitary-adrenal axis, sensitizing the child's depressive pathways and, thus, predisposing him/ her to the development of psychopathologies, including depression ${ }^{6}$.

CONCLUSIONS: Adverse early attachment experiences can be important in understanding the etiology of personality traits and have an impact on further development of psychopathology, particularly depression and anxiety, highlighting the desirability of taking the attachment history into account in clinical practice. Future research should be directed at clarifying the mechanisms of these relationships and investigating the utility of integrating attachment-based assessments and interventions in psychiatric care. 